\title{
Expression and clinical significance of C14orf166 in esophageal squamous cell carcinoma
}

\author{
YAN-WU ZHOU ${ }^{1}$, RONG $^{2}{ }^{2}$, CHAO-JUN DUAN ${ }^{3}$, YANG GAO $^{1}$, \\ YUAN-DA CHENG ${ }^{1}$, ZHI-WEI HE ${ }^{1}$, JUN-XIAN ZENG ${ }^{4}$ and CHUN-FANG ZHANG ${ }^{1}$

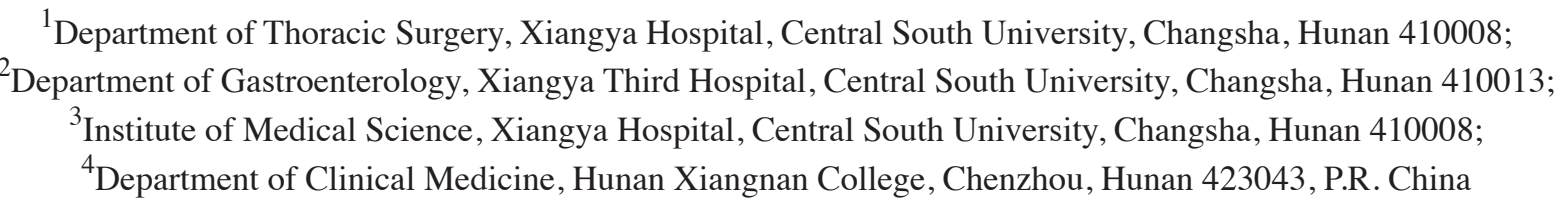

Received November 4, 2015; Accepted November 4, 2016

DOI: $10.3892 / \mathrm{mmr} .2016 .6056$

\begin{abstract}
C14orf166, a $28 \mathrm{kD}$ protein regulating RNA transcription and translation, may serve a critical role in oncogenesis. The aim of the current study was to explore the association between C14orf166 expression and esophageal squamous cell carcinoma (ESCC) and to draw attention to the association between C14orf166 and the initiation, progression and prognosis of ESCC. C14orf166 expression in ESCC and paired normal tissues was detected by immunohistochemical staining, western blotting and reverse transcription-quantitative polymerase chain reaction, and the association between C14orf166 expression and clinicopathological characters of ESCC was analyzed. Survival analysis was used to assess the prognostic significance of C14orf 166 and it was observed that C14orf166 expression was higher in the ESCC tissues when compared with adjacent non-cancerous tissues at protein $(\mathrm{P}<0.001)$ and mRNA levels $(\mathrm{P}<0.001)$. There was a significant difference in $\mathrm{T}$ stage, lymph node metastasis and TNM stage in patients categorized according to different C14orf166 expression levels. The overexpression of C14orf166 was associated with a shorter overall survival and disease-free survival, and multivariate analysis indicated that C14orf166 was an independent prognostic indicator. The present study indicates that the expression of C14orf166 is elevated in ESCC, and is potentially a valuable prognostic predictor for ESCC.
\end{abstract}

\section{Introduction}

As one of the most common types of cancer worldwide, esophageal cancer ranks seventh in incidence and sixth in

Correspondence to: Professor Chun-Fang Zhang, Department of Thoracic Surgery, Xiangya Hospital, Central South University, 87 Xiangya Road, Changsha, Hunan 410008, P.R. China

E-mail: zcf6636169@sina.com

Key words: C14orf166, esophageal squamous cell carcinoma, lymph node metastasis, prognosis leading cause of cancer-associated mortality (1), occurring with an incidence that varies by $\sim 300$-fold worldwide. China and central Asia are represented with the highest rates, particularly in certain counties of China bordering Henan, Hebei and part of Shanxi province; the mortality is as high as $1,100 / 100,000(2-4)$.

Esophageal squamous cell carcinoma (ESCC) is the dominant pathological type, affecting males more often than females (5). Its etiology involves several factors, including genetic, environmental, dietary and hereditary influences (2). Early stage ESCC clinical symptoms and signs are atypical and difficult to detect, thus presenting challenges for diagnosis. In addition, the progression from early to late stage ESCC may be rapid, due to the fact that the esophagus lacks serosa and is adjacent to important organs including the trachea and aorta. At present, the widely recommended management of ESCC is the combined strategy of surgical excision and concurrent chemoradiotherapy, however efficacy remains unsatisfactory with a low 5 -year survival rate and a high prevalence of invasion and metastasis after treatment (6). Therefore, it is important for the control and treatment of ESCC to identify and characterize clinically applicable tumor-specific molecular biomarkers for early detection and targeted prevention. Traditionally, the clinical staging system and pathological standards were used to predict clinical outcome in ESCC, however these are of limited value. Unlike other squamous cell carcinomas, a large proportion of ESCC progresses to metastasis and the dissemination of cancer cells, thus novel targets for clinical intervention are required.

Chromosome 14 open reading frame 166 (C14orf166) is a $28 \mathrm{kD}$ protein whose conserved gene is located on chromosome 14 at 14q22.1, and a transcriptional regulator associated with the repression of the centrosome architecture (7). C14orf166 was first identified as an influenza A virus-associated protein; it can promote viral RNA replication and transcriptional activation by positively modulating host RNA polymerase (RNAP) II and viral RNAP activities. The interaction between C14orf166 and the virus polymerase complex is essential for this function $(8,9)$. Additionally, C14orf 166 has been reported to be a host protein that interacts with hepatitis $\mathrm{C}$ virus during mRNA metabolism and affects host cellular function (10). C14orf166 was also observed to modulate transcription and 
translation by interacting with various transactivators $(11,12)$. It is regarded as a shuttling protein that transports RNAs between the nucleus and cytoplasm, and serves a prominent role in RNA fate and selective gene expression. It has been demonstrated that the C14orf166-DDX1-HSPC117-FAM98B complex helps RNAs shuttle back and forth (13). C14orf166 has also been demonstrated to be a binding partner of Janus kinase (JAK) 2, which can activate excessive signal transducer and activator of transcription (STAT) 3 function to unbalance its tumor promotion and anti-tumor function, hence initiating tumorigenesis $(14,15)$. An increasing number of studies have demonstrated that C14orf166 overexpression was identified in a variety of malignant tumor tissues compared with their paired adjacent non-cancerous tissues, including pancreatic cancer, brain tumor, cervical carcinoma and nasopharyngeal carcinoma. Furthermore, C14orf166 has been regarded as a potential serum biomarker for early diagnosis of tumors, in addition to a predictor for poor prognosis (16-19). However, C14orf166 expression and its role in ESCC require further analysis.

In the present study, C14orf166 expression was detected by immunohistochemistry, western blotting and reverse transcription-quantitative polymerase chain reaction (RT-qPCR) on ESCC tissues, adjacent non-cancerous tissues and several esophageal cancer cell lines respectively. The association between C14orf166 expression and the clinicopathological characteristics of ESCC were analyzed, highlighting the association between C14orf166 overexpression and the occurrence, development and prognosis of ESCC.

\section{Materials and methods}

Clinical samples and cell lines. The 100 patients enrolled in the current study (83 males and 17 females) were recruited from the Department of Thoracic Surgery (Xiangya Hospital; Central South University, Changsha, China) between January 2010 and September 2012, and had been diagnosed with ESCC by three pathologists. The age of the patients ranged from 41 to 74 years and the median age was 58.7 years. They had no history of previous malignancies, and had not previously received chemotherapy, radiotherapy or other treatments before being sampled; the clinical and pathological data were complete and reliable. Tumor differentiation and staging was classified according to the 7th edition of TNM classification of Union Internationale Contra Cancrum (20). Informed consent was acquired from all patients before surgery and the present study was approved by the Ethics Committee of Xiangya Hospital, Central South University. The use of the information and specimens collected has been handled and anonymized according to the ethical and legal standards.

ESCC tissues and paired adjacent normal tissues (greater than $5 \mathrm{~cm}$ away from the tumor margin) were obtained from those patients by esophagus resection. All specimens were immediately snap-frozen in liquid nitrogen and stored at $-80^{\circ} \mathrm{C}$ until RNA and total protein extraction could be performed.

The subjects were followed-up every 3 months during the first postoperative year and for a minimum of 6 months afterwards for survival and recurrence inquiry until death or until the end of the investigation.
Human ESCC cell lines (TE-1, EC109 and EC9706) were obtained from the Cell Bank of the Chinese Academy of Sciences (Shanghai, China). A normal human esophageal epithelial cell line (HEEpic) was purchased from the American Type Culture Collection (Manassas, VA, USA).

$R T$-qPCR. Total RNA was extracted from tissues or cells using TRIzol (Invitrogen; Thermo Fisher Scientific, Inc., Waltham, MA, USA) according to the manufacturer's protocol. After treatment with the DNA-free kit (Ambion; Thermo Fisher Scientific, Inc.) to remove the chromosomal DNA, the complementary DNA was synthesized using the GoScript RT kit (catalog no. A5001; Promega Corporation, Madison, WI, USA) and stored at $-20^{\circ} \mathrm{C}$ until use. The mRNA expression levels of C14orf166 and $\beta$-actin were determined by RT-qPCR using the ABI PRISM 7500 sequence detector system (Applied Biosystems; Thermo Fisher Scientific, Inc.). The primer sequences were sense/anti-sense: C14orf166: 5'-TGCATTGTC AGCAGTTTTTGA-3'/5'-TGACTGGCTTCTTGGTTTAGC-3'; and $\beta$-actin: 5'-GCACCACACCTTCTACAATGAG-3'/5'-GAT AGCACAGCCTGGATAGCA-3'. The mRNA expression levels of the target genes were normalized to the $\beta$-actin signal. All the reactions were conducted in triplicate using $20 \mu$ l samples containing $50 \mathrm{ng}$ complementary DNA. The reaction protocol involved heating for $10 \mathrm{~min}$ at $95^{\circ} \mathrm{C}$, followed by 40 cycles of amplification $\left(15 \mathrm{sec}\right.$ at $95^{\circ} \mathrm{C}$ and $1 \mathrm{~min}$ at $60^{\circ} \mathrm{C}$ ), and a final extension step of $15 \mathrm{sec}$ at $95^{\circ} \mathrm{C}$ and $15 \mathrm{sec}$ at $60^{\circ} \mathrm{C}$. The data were analyzed using the ABI PRISM 7500 Sequence Detection software. The expression of C14orf166 was described as $2^{-\Delta \Delta \mathrm{Cq}}(21)$.

Western blot analysis. The cells and tumor tissues were collected and lysed on ice. Subsequent to centrifugation at 12,000 x $g$ for $20 \mathrm{~min}$, the concentration of proteins was measured and protein samples were denatured by boiling for $10 \mathrm{~min}$, then were and loaded onto a $10 \%$ SDS-PAGE gel for electrophoresis. The proteins were transferred onto a PVDF membrane (EMD Millipore, Billerica, MA, USA) which was then incubated in the blocking solution at room temperature for $2 \mathrm{~h}$. Anti-C14orf166 (1:100; catalog no. 19848-1-AP; ProteinTech Group, Inc., Chicago, IL, USA) and anti-GAPDH (1:5,000; catalog no. MAB5718; R\&D Systems, Inc., Minneapolis, MN, USA) were used for western blotting, incubated at $4^{\circ} \mathrm{C}$ overnight. The membranes were subsequently incubated with horseradish peroxidase (HRP)-labeled goat anti-rabbit IgG (1:6,000; catalog no. NB730-H; Novus Biologicals, LLC, Littleton, $\mathrm{CO}$, USA) or HRP-labeled goat anti-mouse $\operatorname{IgG}(1: 1,000$; catalog no. HAF007; Novus Biologicals, LLC), for $1.5 \mathrm{~h}$ at room temperature. Protein expression was normalized against GAPDH expression. Bands were visualized with the BeyoECL Plus Detection System (Beyotime Institute of Biotechnology, Haimen, China) and Bio-Rad ImageLab software version 3.0 (Bio-Rad Laboratories, Inc., Hercules, CA, USA).

Immunohistochemistry staining. The rapid PV two-step staining method used the following specifications: Paraffin sections of $5 \mu \mathrm{m}$ were obtained, which were heated at $65^{\circ} \mathrm{C}$ for $60 \mathrm{~min}$, then dewaxed in xylene and rehydrated via an ethanol series. Subsequently, high-temperature antigen retrieval was conducted using a microwave in $0.1 \mathrm{M}$ citrate solution ( $\mathrm{pH}$ 6.0) 
for $10 \mathrm{~min}$, followed by incubation in $3 \% \mathrm{H}_{2} \mathrm{O}_{2}$ at room temperature for $20 \mathrm{~min}$. The sections were then incubated in goat serum at room temperature for $20 \mathrm{~min}$, then incubated with anti-C14orf166 rabbit polyclonal antibody (1:100) at $4^{\circ} \mathrm{C}$ overnight. Subsequently, incubation with the secondary anti-rabbit antibody was conducted for $20 \mathrm{~min}$ at room temperature, prior to staining with $\mathrm{DAB} /$ hematoxylin, mounting and examination under the microscope.

Immunohistochemical (IHC) staining was scored independently by two pathologists who had no knowledge of the patients' history or condition and any discrepancy was solved by a consensus. The score of immunoreactivity was obtained by calculating the extent and intensity of the staining of the cells in a semi-quantitative manner. As described previously (22), the standards for evaluation included the following: Positive stain intensity ( 0 , negative; 1 , weak positive; 2 , moderate positive; 3 , strong positive) and proportion of positive areas $(\leq 10 \%=1,10-50 \%=2, \geq 50 \%=3)$. The staining score was the multiplication of the two previous scores. A total of five high power fields in each specimen were selected randomly with the final score as an average of the five scores. Samples were classified as negative when the final scores were 0-3 and positive when $>4$. C14orf166 expression was considered high when the intensity of staining in the tumor cell nuclei was stronger than that of the non-tumorous part and detection of normal esophageal mucosa served as the negative control.

Statistical analysis. SPSS software (version 16.0; SPSS, Inc., Chicago, IL, USA) was used for statistical analyses. All data were presented as the means \pm standard deviation. Categorical variables were compared by the $\chi^{2}$ test and continuous variables were compared using independent two sample $t$-test. Multivariate analyses were performed by the Cox proportional hazard model. Survival curves were performed by the Kaplan-Meier method (the log-rank test). All tests were two-tailed and $\mathrm{P}<0.05$ was considered to indicate a statistically significant difference.

\section{Results}

C14orf166 was overexpressed in human ESCC tissues and cell lines. In the present study, in order to investigate the expression of C14orf166 in ESCC, C14orf166 protein was detected by IHC staining and western blotting. According to IHC staining results, only weak or no immunoreactivity was observed in the adjacent normal tissues, whereas C14orf166 protein was demonstrated to be expressed highly in ESCC tissues, predominantly localized in the carcinoma cell nuclei with cytoplasmic reaction occasionally displayed (Fig. 1A). According to the aforementioned standards, the positive expression rate of C14orf166 protein was $90 \%$ (90/100) in the ESCC group, while it was $14 \%(14 / 100)$ in the paired normal tissues group, a difference that is statistically significant $(\mathrm{P}<0.001$; Fig 1A), this tendency was verified by western blot analysis $(\mathrm{P}<0.00$; Fig. 1D). Furthermore, when the C14orf166 mRNA expression in the 60 cases of ESCCs and the paired normal tissues was analyzed by RT-qPCR, there was a similar trend of C14orf166 expression in the mRNA level observed, compared with the paired adjacent normal tissues; a 2.3-fold increase for C14orf166 expression was noted in ESCC tissues
Table I. C14orf166 expression levels and clinicopathological features in 100 cases of esophageal squamous cell carcinoma.

\begin{tabular}{|c|c|c|c|c|}
\hline \multirow{2}{*}{$\begin{array}{l}\text { Clinicopathological } \\
\text { variable }\end{array}$} & \multirow[b]{2}{*}{$\mathrm{n}$} & \multicolumn{2}{|c|}{$\begin{array}{l}\text { C14orf } 166 \\
\text { expression }\end{array}$} & \multirow[b]{2}{*}{ P-value } \\
\hline & & Low & High & \\
\hline \multicolumn{5}{|l|}{ Age (years) } \\
\hline$\leq 60$ & 54 & 17 & 37 & \\
\hline$>60$ & 46 & 7 & 39 & 0.058 \\
\hline \multicolumn{5}{|l|}{ Gender } \\
\hline Female & 16 & 2 & 14 & \\
\hline Male & 84 & 22 & 62 & 0.239 \\
\hline \multicolumn{5}{|l|}{ Drinking history } \\
\hline Yes & 37 & 9 & 28 & \\
\hline No & 63 & 15 & 48 & 0.954 \\
\hline \multicolumn{5}{|l|}{ Tumor size $(\mathrm{cm})$} \\
\hline$\leq 4$ & 56 & 17 & 39 & \\
\hline$>4$ & 44 & 7 & 37 & 0.093 \\
\hline \multicolumn{5}{|l|}{ Differentiation } \\
\hline Well & 27 & 6 & 21 & \\
\hline Moderate & 49 & 14 & 35 & \\
\hline Poor & 24 & 4 & 20 & 0.518 \\
\hline \multicolumn{5}{|l|}{ T Stage } \\
\hline $\mathrm{T} 1$ & 35 & 15 & 20 & \\
\hline $\mathrm{T} 2$ & 14 & 7 & 7 & \\
\hline $\mathrm{T} 3$ & 39 & 1 & 38 & \\
\hline $\mathrm{T} 4$ & 12 & 1 & 11 & $<0.001$ \\
\hline \multicolumn{5}{|l|}{ N Stage } \\
\hline N0 & 28 & 18 & 10 & \\
\hline N1 & 42 & 3 & 39 & \\
\hline $\mathrm{N} 2$ & 21 & 2 & 19 & \\
\hline N3 & 9 & 1 & 8 & $<0.0001$ \\
\hline \multicolumn{5}{|l|}{ TNM Stage } \\
\hline I & 25 & 21 & 4 & \\
\hline II & 25 & 2 & 23 & \\
\hline III & 50 & 1 & 49 & $<0.0001$ \\
\hline \multicolumn{5}{|l|}{$\begin{array}{l}\text { Adjuvant therapy } \\
\text { following operation }\end{array}$} \\
\hline Yes & 44 & 8 & 36 & \\
\hline No & 56 & 16 & 40 & 0.227 \\
\hline
\end{tabular}

C14orf166, chromosome 14 open reading frame 166.

(Fig. 1B). Comparative analysis of ESCCs with paired adjacent normal tissues further demonstrated that increased C14orf166 expression [more than 2-fold (namely $\log _{2}$ (fold change) $>1$ )] was observed in $76.66 \%$ cases $(46 / 60)$, suggesting that the overexpression of C14orf166 was a frequent event in human ESCC at mRNA and protein level.

Subsequently, C14orf166 expression in ESCC cell lines and normal esophagus epithelial cell line was detected by RT-qPCR and western blotting. As Fig. 1D and E demonstrate, compared with the expression in normal cells (HEEpic), C14orf166 

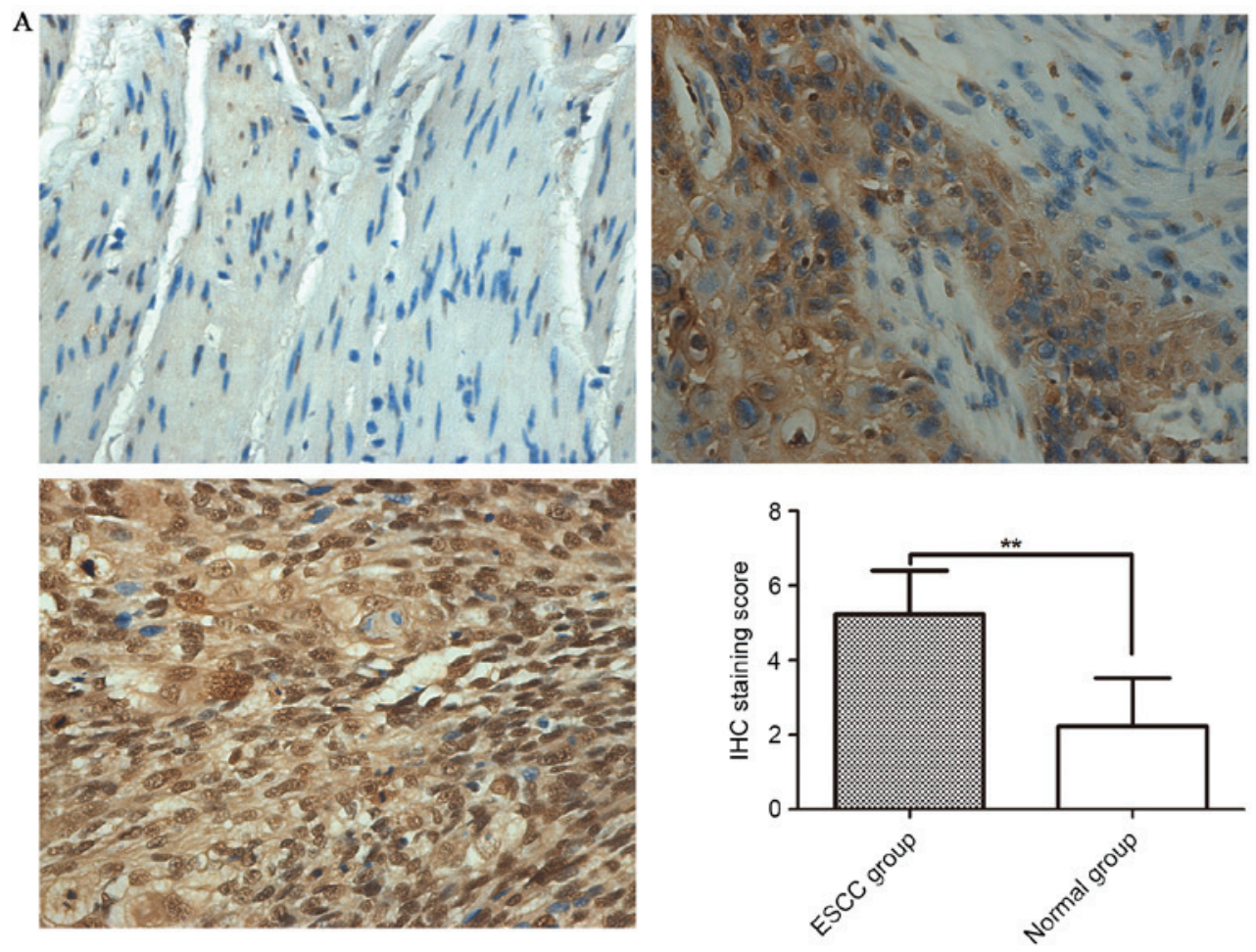

B

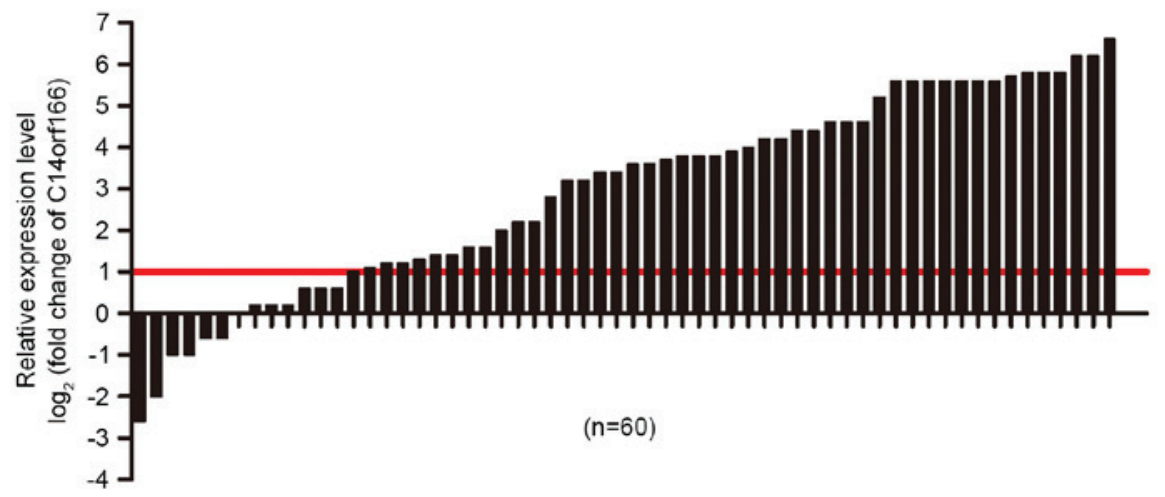

$\mathrm{C}$

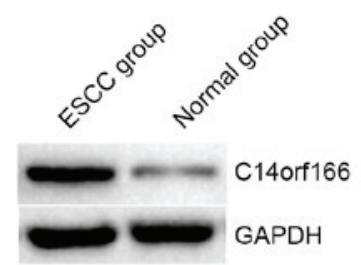

D

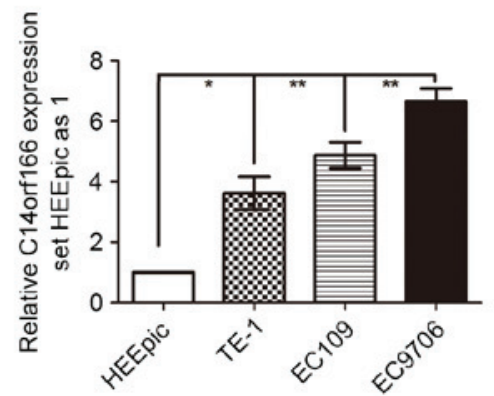

$\mathrm{E}$

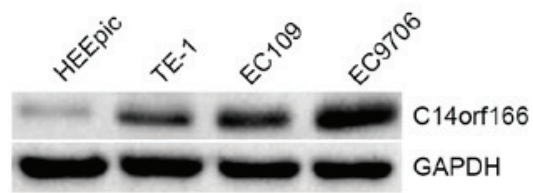

Figure 1. C14orf166 protein and mRNA were frequently upregulated in ESCC tissues and cell lines. (A) Different C14orf166 protein expression levels were detected by IHC staining: Little eIF4E expression was identified in normal esophagus tissues (upper left); moderate C14orf166 expression (upper right), high C14orf166 expression, (lower left). Magnification, $\mathrm{x} 400$. Compared with the adjacent normal tissues, the ESCC tissues were detected to express significantly more C14orf166 in the nuclei ( ${ }^{* *} \mathrm{P}<0.001$ ). (B) Expression of C14orf166 mRNA in 60 pairs of ESCC tissues; expression levels were normalized to the corresponding levels of $\beta$-actin. The relative fold change of mRNA expression was expressed as $2^{\Delta \triangle C q(E S C C / A N C T)}$. (C) Overexpression of C14orf 166 protein in ESCC tissues was verified by western blot analysis. C14orf166 (D) mRNA and (E) protein expression were elevated in esophagus squamous cell carcinoma cell lines. C14orf166, chromosome 14 open reading frame 166; ESCC, esophageal squamous cell carcinoma; IHC, immunohistochemical; ANCT, adjacent non-cancerous tissue. 
A

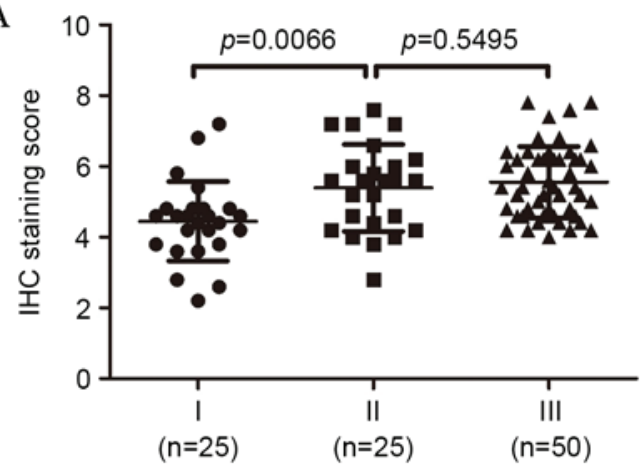

C

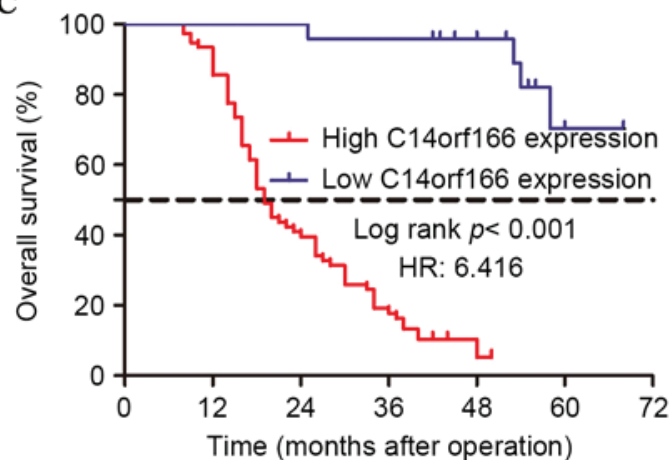

B

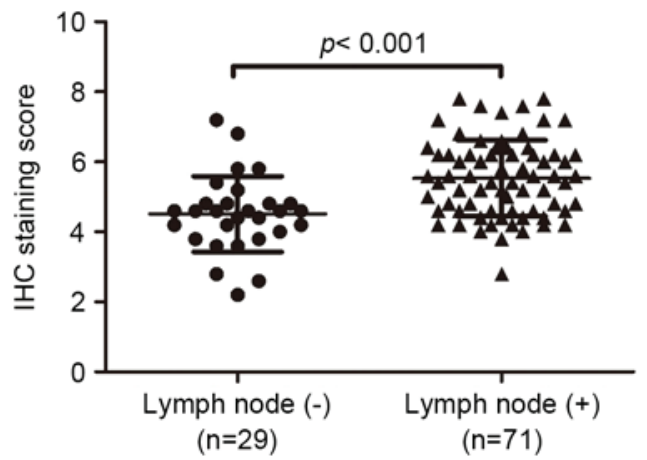

D

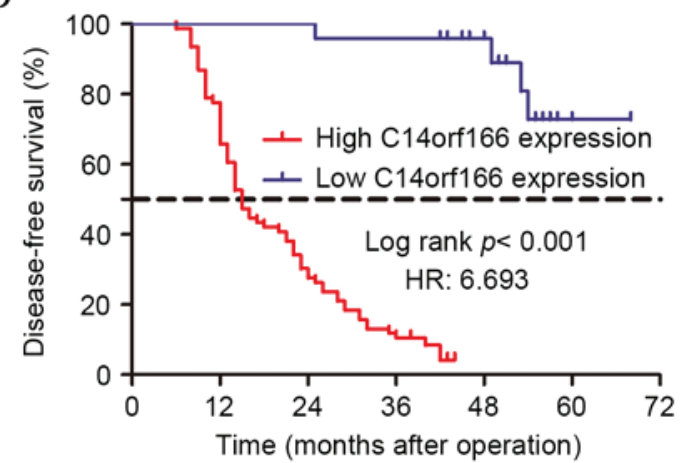

Figure 2. Overexpressed C14orf166 was associated with tumor progression and poor prognosis. According to the IHC staining score, the ESCC group was classified into low $(\mathrm{n}=24)$ and high $(\mathrm{n}=76)$ C14orf166 expression groups, the level of C14orf166 expression was significantly associated with different (A) TNM-stage and (B) lymph nodal involvement, respectively. Kaplan-Meier survival analysis of C14orf166 expression in ESCCs for (C) overall survival and (D) disease-free survival. The higher expression of C14orf166 indicated a poorer disease-free survival $(\mathrm{P}<0.001)$ and overall survival $(\mathrm{P}<0.001)$. $\mathrm{C} 14$ orf 166 , chromosome 14 open reading frame 166; IHC, immunohistochemical; ESCC, esophageal squamous cell carcinoma.

mRNA and protein expression levels were elevated significantly in human ESCC cell lines (TE-1, EC109 and EC9706).

Elevated C14orf166 expression was associated with clinicopathological characteristics in ESCC. The association between C14orf166 expression and the clinicopathological features of ESCC was explored by the $\chi^{2}$ test. As summarized in Table I, the high expression of C14orf166 was significantly associated with $\mathrm{T}$ staging $(\mathrm{P}<0.001)$, lymph node metastasis $(\mathrm{P}=0.000)$ and $\mathrm{TNM}$ stage $(\mathrm{P}=0.000)$ respectively. However, no significant association was observed between C14orf166 expression and variables including age $(\mathrm{P}=0.058)$, gender $(\mathrm{P}=0.239)$, drinking history $(\mathrm{P}=0.954)$, tumor size $(\mathrm{P}=0.093)$ or tumor differentiation $(\mathrm{P}=0.518)$. Subgroup analysis indicated that overexpressed C14orf166 was associated with advanced stage and lymph node metastasis (Fig. 2A and B). As presented in Table I, no statistical difference was identified between C14orf166 overexpression and the success of subsequent adjuvant therapy following the operation, which was likely affected by multiple factors, including disease degree and family income. In addition, in the 44 patients in the study who received chemotherapy or radiotherapy, no association between the increased C14orf166 expression and therapeutic effect was identified $(\mathrm{P}=0.227)$.

Overexpression of C14orf166 was associated with the poor prognosis of ESCC. To assess the role of C14orf166 expression in ESCC prognosis, the 100 patients were followed up subsequent to the operations. Kaplan-Meier survival analysis and the log-rank test were performed and demonstrated that a high level of C14orf166 expression is closely associated with a shorter overall survival $(\mathrm{OS})$ time $(\mathrm{P}<0.001)$ and disease-free survival (DFS) time $(\mathrm{P}<0.001)$; the patients with low C14orf166 expression had an approximately 3-fold longer OS time and 4-fold longer DFS time than those with overexpressed C14orf166.

To validate the feasibility of C14orf166 expression in ESCC prognosis, the Cox proportional hazards regression model was introduced. Multivariate survival analysis on all characteristics demonstrated that survival time was significantly dependent on lymph node involvement, TNM stage and C14orf166 expression level (Table II), they were independent prognostic factors for OS and DFS, indicating that C14orf166 may be a potential clinical prognostic predicator for patients with ESCC.

\section{Discussion}

As a constituent of nuclear and cytoplasmic protein complexes, C14orf166 is located in the nuclear and cytoplasm and, partly as a result of this location, it has been identified to be a protein serving a vital role in cellular RNA expression regulation, including RNA transcription, maturation and translation. C14orf166 participates in RNAP II-directed RNA transcription by interacting with the RNAP II directly, aiding to shuttle important elements. It has also been reported to transport specific mRNAs from the cell body to the dendrites in the developing brain, which additionally implies a significant role in brain development (23). Functions of C14orf166 account 
Table II. Cox regression multivariate analysis of overall and disease-free survival in 100 patients with esophageal squamous cell carcinoma.

\begin{tabular}{|c|c|c|c|c|c|}
\hline \multirow[b]{2}{*}{ Variable } & \multirow[b]{2}{*}{$\mathrm{n}$} & \multicolumn{2}{|c|}{ Overall survival } & \multicolumn{2}{|c|}{ Disease-free survival } \\
\hline & & HR $(95 \%$ CI $)$ & P-value & $\mathrm{HR}(95 \% \mathrm{CI})$ & P-value \\
\hline \multicolumn{6}{|l|}{ Age (years) } \\
\hline$\leq 60$ & 54 & 1 & & 1 & \\
\hline$>60$ & 46 & $0.79(0.42-1.51)$ & 0.483 & $0.73(0.39-1.35)$ & 0.313 \\
\hline \multicolumn{6}{|l|}{ Gender } \\
\hline Female & 16 & 1 & & 1 & \\
\hline Male & 84 & $0.75(0.36-1.58)$ & 0.449 & $0.60(0.28-1.30)$ & 0.198 \\
\hline \multicolumn{6}{|l|}{ Drinking history } \\
\hline Yes & 37 & 1 & & 1 & \\
\hline No & 63 & $1.14(0.61-2.13)$ & 0.683 & $1.36(0.74-2.52)$ & 0.320 \\
\hline \multicolumn{6}{|c|}{ Tumor size $(\mathrm{cm})$} \\
\hline$\leq 4$ & 56 & 1 & & 1 & \\
\hline$>4$ & 44 & $1.43(0.81-2.53)$ & 0.218 & $1.64(0.95-2.83)$ & 0.074 \\
\hline Differentiation & & & 0.930 & & 0.809 \\
\hline Well & 27 & 1 & & 1 & \\
\hline Moderate & 49 & $0.97(0.48-1.99)$ & 0.942 & $1.24(0.63-2.45)$ & 0.532 \\
\hline Poor & 24 & $0.87(0.39-1.95)$ & 0.737 & $1.11(0.51-2.41)$ & 0.794 \\
\hline T stage & & & 0.620 & & 0.254 \\
\hline $\mathrm{T} 1$ & 35 & 1 & & 1 & \\
\hline $\mathrm{T} 2$ & 14 & $1.88(0.67-5.28)$ & 0.233 & $2.53(0.91-7.00)$ & 0.075 \\
\hline $\mathrm{T} 3$ & 39 & $1.56(0.45-5.39)$ & 0.482 & $1.77(0.55-5.75)$ & 0.339 \\
\hline $\mathrm{T} 4$ & 12 & $2.42(0.32-18.08)$ & 0.391 & $3.18(0.43-23.71)$ & 0.260 \\
\hline $\mathrm{N}$ stage & & & 0.023 & & 0.032 \\
\hline N0 & 28 & 1 & & 1 & \\
\hline N1 & 42 & $2.41(0.42-13.67)$ & 0.322 & $2.16(0.44-10.62)$ & 0.345 \\
\hline $\mathrm{N} 2$ & 21 & $3.50(0.45-27.28)$ & 0.231 & $3.81(0.56-25.94)$ & 0.172 \\
\hline N3 & 9 & $46.19(3.13-681.5)$ & 0.005 & $20.19(1.86-218.74)$ & 0.013 \\
\hline TNM stage & & & 0.004 & & 0.006 \\
\hline I & 25 & 1 & & 1 & \\
\hline II & 25 & $1.93(0.24-15.64)$ & 0.537 & $2.66(0.35-20.39)$ & 0.348 \\
\hline III & 50 & 36.85 (1.51-900.7) & 0.027 & $42.89(1.83-1003.01)$ & 0.019 \\
\hline \multicolumn{6}{|c|}{ C14orf166 expression } \\
\hline Low & 24 & 1 & & 1 & \\
\hline High & 76 & $16.96(1.89-152.41)$ & 0.012 & $20.33(2.25-183.34)$ & 0.007 \\
\hline
\end{tabular}

HR, hazard ratio; CI, confidence intervals; C14orf166, chromosome 14 open reading frame 166.

for normal RNA transcription, mRNA expression, signal transduction, and important cellular phenotypes including proliferation, apoptosis and differentiation. Blocking C14orf166 will reduce mRNA translation by $50 \%$, severely impairing cellular activity (12). However, there are also genes associated with oncogenesis and tumor invasion behavior under the regulation of C14orf166, and overexpression of C14orf166 was identified in multiple tumor tissues relative to its adjacent normal tissues (16-19).

To the best of our knowledge, there have been no investigations into a potential role for C14orf166 in ESCC at present and this is the first study on the possible association between C14orf166 and ESCC. The present study investigated the protein and mRNA expression of C14orf166 in a series of paired ESCC specimens with intact follow-up data. C14orf166 was first identified to be consistently upregulated in ESCC tissues and cell lines. Consistent with the expression in other carcinoma tissues, IHC staining demonstrated that C14orf166 protein expression was clearly elevated in ESCC tissues, as demonstrated by western blotting. RT-qPCR analysis additionally displayed a high frequency of C14orf166 mRNA overexpression in the majority of ESCC cases, which 
demonstrated C14orf166 was transcriptionally and translationally upregulated in ESCC, indicating that C14orf166 may have a significant role in ESCC initiation.

From the clinical data, C14orf166 expression was correlated with respect to various clinicopathological characteristics in 100 patients with ESCC. As predicted, the high expression of C14orf166 had no correlation with age, gender, drinking history, tumor size or even tumor differentiation, however it demonstrated a strong association with the $\mathrm{T}$ staging, lymph node involvement and TNM stage. This indicated that the higher expression of C14orf166 leads to the more malignant biological characteristics, despite the tumor differentiation; this is consistent with a previous study in pancreatic cancer (18). Additionally, the data produced by the current study demonstrated that elevated C14orf166 expression was a negative prognostic predicator for patients with ESCC. As an adjuvant therapy, concurrent radiochemotherapy is used clinically to improve the prognosis of patients with ESCC, while patients undergoing adjuvant therapy experience a higher frequency of severe side effects including oral mucous ulceration and vomiting. Thus, C14orf166 may represent a potential biomarker to guide the selection of therapeutic strategy and improve survival, although those who received adjuvant therapy in the present study could not demonstrate this advantage, which was limited by the small size of the study. Furthermore, C14orf166 has been reported to be detected in the serum of pancreatic carcinoma (13), which indicates a potential novel biomarker for early diagnosis and an aid in therapy design. Therefore, further investigations are required to fully elucidate this potential biomarker.

In addition to previous studies, the results of the present study indicate that C14orf166 may participate in oncogenesis and tumor progression, which may be partly explained by the fact that, in addition to regulation of mRNA expression, C14orf166 serves a vital role in signal transduction during cell division; protein correlation profiling analysis in diverging cells demonstrated that C14orf166 interplayed with ninein in the centrosomes (24), which was confirmed by Howng et al (25) whose research demonstrated that $\mathrm{C} 14$ orf166 could bind to the ninein, thus inhibiting phosphorylation by glycogen synthase kinase $3 \beta$ (GSK-3 $\beta$ ). GSK-3 $\beta$ can phosphorylate a variety of substrates participating in signal transduction and cell proliferation, in addition to organ development. Dysfunction of GSK- $3 \beta$ is prevalent in tumor formation, promoting progression and contributing to drug resistance by interfering with signaling pathways, including phosphatidylinositol-3-kinase (PI3K)-protein kinase $\mathrm{B}(\mathrm{AKT})$-mammalian target of rapamycin (mTOR), Wnt/ $\beta$-catenin and JAK2/STAT3 signaling pathways (26-28). Abnormal expression of PI3K-AKT-mTOR is frequent in melanoma and indicates a poor prognosis, and thus inhibition of the mTOR pathway will benefit numerous patients in the clinic (29). Ge et al (30) demonstrated that through abnormal activation of Wnt/ $\beta$-catenin pathway, miR-942 aided to maintain cancer stem cell-like traits in ESCC, inducing poor prognosis and unsatisfactory drug effects. In addition, GSK-3 $\beta$ can promote esophagus carcinoma cells metastasis and spread by degrading $\beta$-catenin $(31,32)$. The dysregulated intracellular JAK2/STAT3 signaling pathway is common in numerous types of carcinoma and is associated with malignancy and poor prognosis, an antagonist of the pathway attenuates tumor bearing and improves drug efficacy $(33,34)$. Zhang et al (19) identified that C14orf166 overexpression was associated with lymph node involvement and shorter OS and DFS in cervical cancer, which was associated with abnormal JAK2/STAT3 pathway activity. Chen et al (35) reported that C14orf166, together with acylglycerol kinase, were identified as JH2-interacting proteins, which consecutively activated the JAK2/STAT3 signaling pathway to promote esophageal squamous cell generation and enhanced the cancer stem cell population. A JAK2 inhibitor will block the growth of the ESCC through the JAK/STAT3 pathway (36). The current study hypothesized that influenced by this signaling pathway, C14orf166 modifies the downstream signal transduction via GSK-3 $\beta$, affecting the constitution and stability of centrosomes, and excessive C14orf166 expression promotes gene translation, resulting in tumor origination and progression.

Taken together, these data in the present study suggest that C14orf166 may serve a vital role in the formation and progression of ESCC. C14orf166 may be a potential biomarker for lymph node metastasis and poor prognosis in ESCC, which may aid in the identification of patients at high risk and offer a rationale for selecting appropriate treatment. Although the present study shows preliminary data for the potential association between C14orf166 overexpression and ESCC, further investigation is required to fully elucidate the underlying mechanism of C14orf166 in regulating the oncogenesis, progression, metastasis and prognosis of ESCC.

\section{Acknowledgements}

The current study was supported by the National Natural Scientific Foundation of China (grant no. 81372515) and the Science Funds for Young Scholar of Xiangya Hospital (grant no. 2013Q02).

\section{References}

1. Torre LA, Bray F, Siegel RL, Ferlay J, Lortet-Tieulent J and Jemal A: Global cancer statistics, 2012. CA Cancer J Clin 65: 87-108, 2015.

2. Guohong Z, Min S, Duenmei W, Songnian H, Min L, Jinsong L, Hongbin L, Feng Z, Dongping T, Heling Y, et al: Genetic heterogeneity of oesophageal cancer in high-incidence areas of southern and northern China. PLoS One 5: e9668, 2010.

3. Jemal A, Murray T, Samuels A, Ghafoor A, Ward E and Thun MJ: Cancer statistics, 2003. CA Cancer J Clin 53: 5-26, 2003.

4. Tran GD, Sun XD, Abnet CC, Fan JH, Dawsey SM, Dong ZW, Mark SD, Qiao YL and Taylor PR: Prospective study of risk factors for esophageal and gastric cancers in the Linxian general population trial cohort in China. Int J Cancer 113: 456-463, 2005.

5. Enzinger PC and Mayer RJ: Esophageal cancer. N Engl J Med 349: 2241-2252, 2003.

6. Siegel R, DeSantis C, Virgo K, Stein K, Mariotto A, Smith T, Cooper D, Gansler T, Lerro C, Fedewa S, et al: Cancer treatment and survivorship statistics, 2012. CA Cancer J Clin 62: 220-241, 2012.

7. Lupi I, Broman KW, Tzou SC, Gutenberg A, Martino E and Caturegli P: Novel autoantigens in autoimmune hypophysitis. Clin Endocrinol (Oxf) 69: 269-278, 2008.

8. Rodriguez A, Pèrez-González A and Nieto A: Cellular human CLE/C14orf166 protein interacts with influenza virus polymerase and is required for viral replication. J Virol 85: 12062-12066, 2011.

9. Natasya Naili MN, Hasnita CH, Shamim AK, Hasnan J, Fauziah MI, Narazah MY, James A, Zulkiflee S, Nidzam MM and Zilfalil BA: Chromosomal alterations in Malaysian patients with nasopharyngeal carcinoma analyzed by comparative genomic hybridization. Cancer Genet Cytogenet 203: 309-312, 2010.

10. Lee JW, Liao PC, Young KC, Chang CL, Chen SS, Chang TT, Lai MD and Wang SW: Identification of hnRNPH1, NF451, and C14orf166 as novel host interacting partners of the mature hepatitis C virus core protein. J Proteome Res 10: 4522-4534, 2011. 
11. Schamel K, Staeheli P and Hausmann J: Identification of the immunodominant $\mathrm{H}-2 \mathrm{~K}(\mathrm{k})$-restricted cytotoxic T-cell epitope in the Borna disease virus nucleoprotein. J Virol 75: 8579-8588, 2001.

12. Huarte M, Sanz-Ezquerro JJ, Roncal F, Ortin J and Nieto A: PA subunit from influenza virus polymerase complex interacts with a cellular protein with homology to a family of transcriptional activators. J Virol 75: 8597-8604, 2001.

13. Pèrez-González A, Pazo A, Navajas R, Ciordia S, Rodriguez-Frandsen A and Nieto A: hCLE/C14orf166 associates with DDX1-HSPC117-FAM98B in a novel transcription-dependent shuttling RNA-transporting complex. PLoS One 9: e90957, 2014.

14. Yu H, Pardoll D and Jove R: STATs in cancer inflammation and immunity: A leading role for STAT3. Nat Rev Cancer 9: 798-809, 2009.

15. Ernst $M$ and Putoczki TL: Stat3: Linking inflammation to (gastrointestinal) tumourigenesis. Clin Exp Pharmacol Physiol 39: 711-718, 2012.

16. Wang J, Gu Y, Wang L, Hang X, Gao Y, Wang $\mathrm{H}$ and Zhang $\mathrm{C}$ : HUPO BPP pilot study: A proteomics analysis of the mouse brain of different developmental stages. Proteomics 7: 4008-4015, 2007.

17. Guo J, Wang W, Liao P, Lou W, Ji Y, Zhang C, Wu J and Zhang S: Identification of serum biomarkers for pancreatic adenocarcinoma by proteomic analysis. Cancer Sci 100: 2292-2301, 2009.

18. Cui Y, Wu J,Zong M, Song G, Jia Q, Jiang J and Han J: Proteomic profiling in pancreatic cancer with and without lymph node metastasis. Int J Cancer 124: 1614-1621, 2009.

19. Zhang W, Ou J, Lei F, Hou T, Wu S, Niu C, Xu L and Zhang Y: C14ORF166 overexpression is associated with pelvic lymph node metastasis and poor prognosis in uterine cervical cancer. Tumour Biol 37: 369-379, 2016.

20. Sobin LH, Gospodarowicz MK and Wittekind C: TNM classification of malignant tumors. 7th edition. Oxford: Wiley-Blackwell, 2010.

21. Livak KJ and Schmittgen TD: Analysis of relative gene expression data using real-time quantitative PCR and the 2(-Delta Delta C (T)) Method. Methods 25: 402-408, 2001.

22. Zhang S, Tang W, Weng S, Liu X, Rao B, Gu J, Chen S, Wang Q, Shen X, Xue R and Dong L: Apollon modulates chemosensitivity in human esophageal squamous cell carcinoma. Oncotarget 5: 7183-7197, 2014.

23. Elvira G, Wasiak S, Blandford V, Tong XK, Serrano A, Fan X, del Rayo Sánchez-Carbente M, Servant F, Bell AW, Boismenu D, et al: Characterization of an RNA granule from developing brain. Mol Cell Proteomics 5: 635-651, 2006.

24. Andersen JS, Wilkinson CJ, Mayor T, Mortensen P, Nigg EA and Mann M: Proteomic characterization of the human centrosome by protein correlation profiling. Nature 426: 570-574, 2003.
25. Howng SL, Hsu HC, Cheng TS, Lee YL, Chang LK, Lu PJ and Hong YR: A novel ninein-interaction protein, CGI-99, blocks ninein phosphorylation by GSK3beta and is highly expressed in brain tumors. FEBS Lett 566: 162-168, 2004.

26. Zhao P, Li Q, Shi Z, Li C, Wang L, Liu X, Jiang C, Qian X, You Y, Liu N, et al: GSK-3 $\beta$ regulates tumor growth and angiogenesis in human glioma cells. Oncotarget 6: 31901-31915, 2015.

27. Gao Y, Liu Z, Zhang X, He J, Pan Y, Hao F, Xie L, Li Q, Qiu X and Wang E: Inhibition of cytoplasmic GSK-3 $\beta$ increases cisplatin resistance through activation of $\mathrm{Wnt} / \beta$-catenin signaling in A549/DDP cells. Cancer Lett 336: 231-239, 2013.

28. Haraguchi K, Ohsugi M, Abe Y, Semba K, Akiyama T and Yamamoto T: Ajuba negatively regulates the Wnt signaling pathway by promoting GSK-3beta-mediated phosphorylation of beta-catenin. Oncogene 27: 274-284, 2008.

29. Kong Y, Si L, Li Y, Wu X, Xu X, Dai J, Tang H, Ma M, Chi Z, Sheng X, et al: Analysis of mTOR gene aberrations in melanoma patients and evaluation of their sensitivity to PI3K-AKT-mTOR pathway inhibitors. Clin Cancer Res 22: 1018-1027, 2016.

30. Ge C, Wu S, Wang W, Liu Z, Zhang J, Wang Z, Li R, Zhang Z, Li Z, Dong S, et al: miR-942 promotes cancer stem cell-like traits in esophageal squamous cell carcinoma through activation of Wnt $/ \beta$-catenin signalling pathway. Oncotarget 6: 10964-10977, 2015.

31. He H, Ding F, Li Y, Luo A, Chen H, Wu C and Liu Z: Migfilin regulates esophageal cancer cell motility through promoting GSK-3 $\beta$-mediated degradation of $\beta$-catenin. Mol Cancer Res 10: 273-281, 2012.

32. Zhang W, Yan S, Liu M, Zhang G, Yang S, He S, Bai J, Quan L, Zhu H, Dong Y and Xu N: Beta-Catenin/TCF pathway plays a vital role in selenium induced-growth inhibition and apoptosis in esophageal squamous cell carcinoma (ESCC) cells. Cancer Lett 296: 113-122, 2010.

33. Mukthavaram R, Ouyang X, Saklecha R, Jiang P, Nomura N, Pingle SC, Guo F, Makale $M$ and Kesari S: Effect of the JAK2/STAT3 inhibitor SAR317461 on human glioblastoma tumorspheres. J Transl Med 13: 269, 2015.

34. Zhao H, Guo Y, Li S, Han R, Ying J, Zhu H, Wang Y, Yin L, Han Y, Sun L, et al: A novel anti-cancer agent Icaritin suppresses hepatocellular carcinoma initiation and malignant growth through the IL-6/Jak2/Stat3 pathway. Oncotarget 6: 31927-31943, 2015.

35. Chen $X$, Ying Z, Lin X, Lin H, Wu J, Li M and Song L: Acylglycerol kinase augments JAK2/STAT3 signaling in esophageal squamous cells. J Clin Invest 123: 2576-2589, 2013.

36. Fang J, Chu L, Li C, Chen Y, Hu F, Zhang X, Zhao H, Liu Z and Xu Q: JAK2 inhibitor blocks the inflammation and growth of esophageal squamous cell carcinoma in vitro through the JAK/STAT3 pathway. Oncol Rep 33: 494-502, 2015. 